\title{
Le regroupement municipal des villes du Saguenay : son objet ultime et sa raison d'être
}

\author{
Sergieh F. Moussally ${ }^{1}$ \\ Université du Québec à Chicoutimi
}

\section{Introduction : la formation de la ville de Saguenay et le choix du référentiel pertinent d'évaluation}

En ordonnant la fusion des villes de Chicoutimi, Jonquière, La Baie, Laterrière, Shipshaw, Lac Kénogami et une partie de Canton Tremblay, le décideur public québécois envisageait la réalisation simultanée de deux objectifs distincts :

a) accroître l'efficacité et la qualité des services municipaux réalisables par le regroupement des trois principales villes de la conurbation du Saguenay;

b) stimuler le développement que la nouvelle entité urbaine peut réaliser en raison de sa taille, l'accroissement des ressources qu'elle peut mobiliser et l'effet de synergie résultant de la fusion de trois entités municipales remplissant des fonctions économiques différenciées et complémentaires, soit :

- l'agglomération industrielle de Jonquière;

- la métropole de localisation des services : Chicoutimi;

- le nœud central de communication de La Baie (maritime, aérien, terrestre et ferroviaire).

De ce fait, toute évaluation des avantages-coûts anticipés de la formation de Saguenay trouve sa validité en subordonnant la réalisation de l'objectif fiscal de rationalisation et d'allégement du fardeau du contribuable pour une qualité donnée de services au but ultime voulu par le décideur québécois, soit la dota- tion de l'agglomération urbaine du Saguenay d'une taille et d'une échelle de moyens permettant la création d'un pôle dynamique de développement.

Réciproquement, un référentiel statique d'évaluation des avantages-coûts de la fusion postulant le maintien de la base économique au Saguenay dans sa configuration actuelle ne peut fournir un éclairage approprié des enjeux réels et prévisibles de la fusion.

Une trajectoire de dégradation du vecteur économique et démographique que Saguenay peut emprunter à moyen ou à long terme en raison d'une turpitude stratégique finirait par annuler tout avantage fiscal réalisable à court terme.

L'objet de cette réflexion à propos des avantagescoûts du regroupement municipal au Saguenay est de rendre compte des perspectives de développement réalisables en raison de l'intégration des trois composantes de la nouvelle entité urbaine générant un effet synergique de complémentarité, d'une part, de l'accroissement de l'envergure et de la diversité des moyens (budgétaire, fiscal, informationnel, réglementaire) que Saguenay peut mobiliser pour transformer ses assises (démographique, technologique et économique), d'autre part.

Plus concrètement, les avantages-coûts du regroupement municipal trouvent leur référentiel pertinent d'estimation en fonction de l'objectif ultime que constitue la création au Saguenay d'un pôle de compétitivité générant une transformation de la base démoéconomique et technique de la nouvelle agglomération. 
Un pôle urbain de compétitivité définit un lieu de localisation d'activités et de grappes industrielles, caractérisé par les données suivantes :

- des externalités (gains) sont réalisables par les entreprises localisées dans le pôle en raison de la qualité de l'infrastructure technique, industrielle et urbaine;

- des réseaux formels et informels d'échanges entre les firmes contribuent à stimuler la productivité, la réduction du risque, l'apprentissage des technologies externes et l'innovation;

- le pôle dispose d'avantages spécifiques de localisation en raison de son infrastructure et des services qu'il peut fournir aux investisseurs externes à la recherche de lieux optimaux;

- les produits du pôle exportés au reste du monde engendrent des gains nets en valeur ajoutée. Ces gains renforcent la dynamique du développement du pôle.

\section{Cadre d'analyse et hypothèse systémique}

La théorie des systèmes liés appliquée aux structures territoriales fournit un cadre approprié d'analyse des modalités concrètes de transformation des assises économiques d'une agglomération, sans égard à sa taille, sa localisation dans l'espace des échanges ou de ses handicaps initiaux spécifiques ${ }^{2}$. Dans le contexte de la mondialisation caractérisé par la mobilité des activités, des technologies et du capital, le postulat fondamental en matière de développement repose sur les gains réalisables sur les échanges avec le monde par tout opérateur économique.

Les agglomérations urbaines disposant d'une latitude de liberté dans le choix de leurs stratégies, d'une dotation appropriée en ressources (fiscale, financière, informationnelle, réglementaire, énergétique, etc.) sont considérées comme des systèmes liés agissant comme tout autre opérateur économique, selon la logique de l'efficacité et de la compétitivité qu'impose la dynamique de la mondialisation. Leur accessibilité à toute trajectoire de développement réalisable dans l'espace des échanges qui les intègrent procède du triptyque suivant :

- déploiement d'une stratégie appropriée d'équipement permettant la création d'un pôle de compé-

titivité opératoire sur les circuits mondiaux des échanges;

- utilisation des leviers de la mondialisation en vue de faire valoir les avantages concurrentiels spécifiques de leur pôle de compétitivité;

- mobilisation des ressources et des moyens financiers requis à la formation d'un pôle de compétitivité, son équipement en infrastructure, son adaptation aux stratégies des grappes et des firmes à la recherche des lieux optimaux de localisation de leurs activités.

Ce schéma de développement repose sur l'envergure et la continuité des gains sur les échanges qu'un système urbain peut réaliser en raison de son efficacité stratégique et attractive des ressources. Par conséquent, contrairement au schéma déterministe du développement qui prévalait durant la seconde révolution industrielle achevée en 1981, les agglomérations urbaines des pays industriels avancés peuvent créer un pôle de compétitivité mondial en faisant valoir une compétence dans l'emploi de leur dotation en ressources.

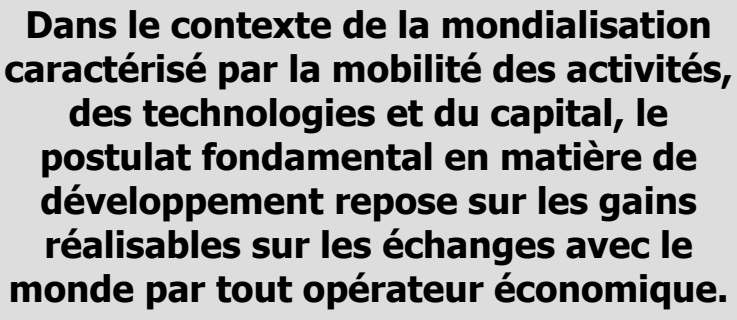

En pratique, la logique sous-jacente à la mondialisation signifie que les handicaps conventionnels des formations urbaines ${ }^{3}$ ne constituent plus des obstacles contraignant au développement. Cette donnée est particulièrement valide dans les formations ayant atteint le stade de la primauté du progrès technique et l'innovation en matière d'efficacité et de compétitivité, comme c'est le cas au Québec. L'hypothèse que nous formulons à propos de l'évaluation des avantagescoûts du regroupement municipal au Saguenay comporte les propositions suivantes :

a) l'accroissement de la taille réalisé par la fusion des trois principales agglomérations du Saguenay (Chicoutimi, Jonquière, La Baie) ouvre une perspective concrète au développement de l'ensemble 
considéré, en fonction de son aptitude à créer un pôle de compétitivité mondiale;

b) l'effet de synergie réalisable de l'intégration des services municipaux de la ville de Saguenay implique la conception et la mise en œuvre d'une stratégie appropriée de transformation des assises économiques de Saguenay. Cette exigence constitue la condition nécessaire au développement de l'agglomération urbaine constituée par la fusion;

c) la condition suffisante est d'ordre instrumental et financier.

\section{La création d'un pôle de compétitivité au Saguenay}

La création d'un pôle de compétitivité opérant sur les marchés dans une agglomération urbaine localisée dans une région ressources requiert un effort soutenu d'investissements portant sur les volets suivants, formant un système synergique de création d'avantages concurrentiels territoriaux (AVCRTR) (figure 1) :

a) Le renforcement de la capacité technologique de l'entité définie comme résultante systémique de l'articulation des composantes suivantes :

- les connaissances scientifiques requises aux activités de développement à localiser;

- les technologies associées aux nouvelles activités à intégrer;

- les industries en segments de grappes intensives en valeur ajoutée à localiser;

- le système urbain d'encadrement, d'orientation et de support en services générant des avantages spécifiques de localisation d'une (ou plusieurs) grappe(s) industrielles(s).

b) Le déploiement d'une stratégie d'insertion dans les réseaux mondiaux (nord-américain) des échanges utilisant plusieurs vecteurs d'insertion :

- les alliances stratégiques entre firmes régionales et entreprises du reste du monde;
- la rétro ingénierie et l'apprentissage des technologies avancées extérieures;

- la participation à des joint-ventures;

- le déploiement des entreprises régionales dans leur zone commerciale

c) L'exploitation des avantages liés à la dotation en ressources de la région de localisation de l'entité urbaine et/ou plus-value (extra) qui résulte de l'écart entre le coût de production dans la région et le prix mondial des biens intensifs en valeur ajoutée qui sont exportés. Le capital mondial et les technologies à la recherche de lieux optimaux de localisation sont attirés par la plus-value réalisable dans les régions ressources caractérisées par un retard d'adaptation des salaires à la croissance de la productivité résultant du progrès technologique ${ }^{4}$.

d) La capacité décisionnelle et opératoire de la structure urbaine d'encadrement, d'orientation et de support à la création sui generis de nouveaux avantages de localisation répondant aux exigences des firmes et des activités à localiser dans l'entité urbaine.

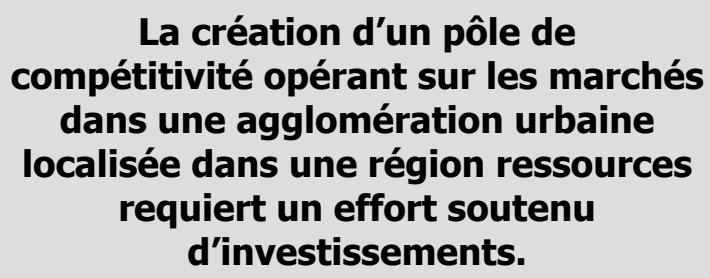

Ce schéma établi par Manuel Castells ${ }^{5}$ s'applique au contexte spécifique qui caractérise l'agglomération urbaine de Saguenay, soit :

- l'insuffisance de la capacité technologique établie;

- les multiples lacunes en matière de formation et de renouvellement de la force de travail spécialisée ou qualifiée;

- la rareté des équipements urbains et ou des terrains disponibles dans les parcs industriels de Saguenay;

- les déficiences du réseau de communication, et en particulier la capacité limitée du port de Saguenay (Grande-Anse). 
Figure 1 - La stratégie de création d'un pôle de compétitivité au Saguenay

\section{Les leviers des avantages de localisation à exploiter}

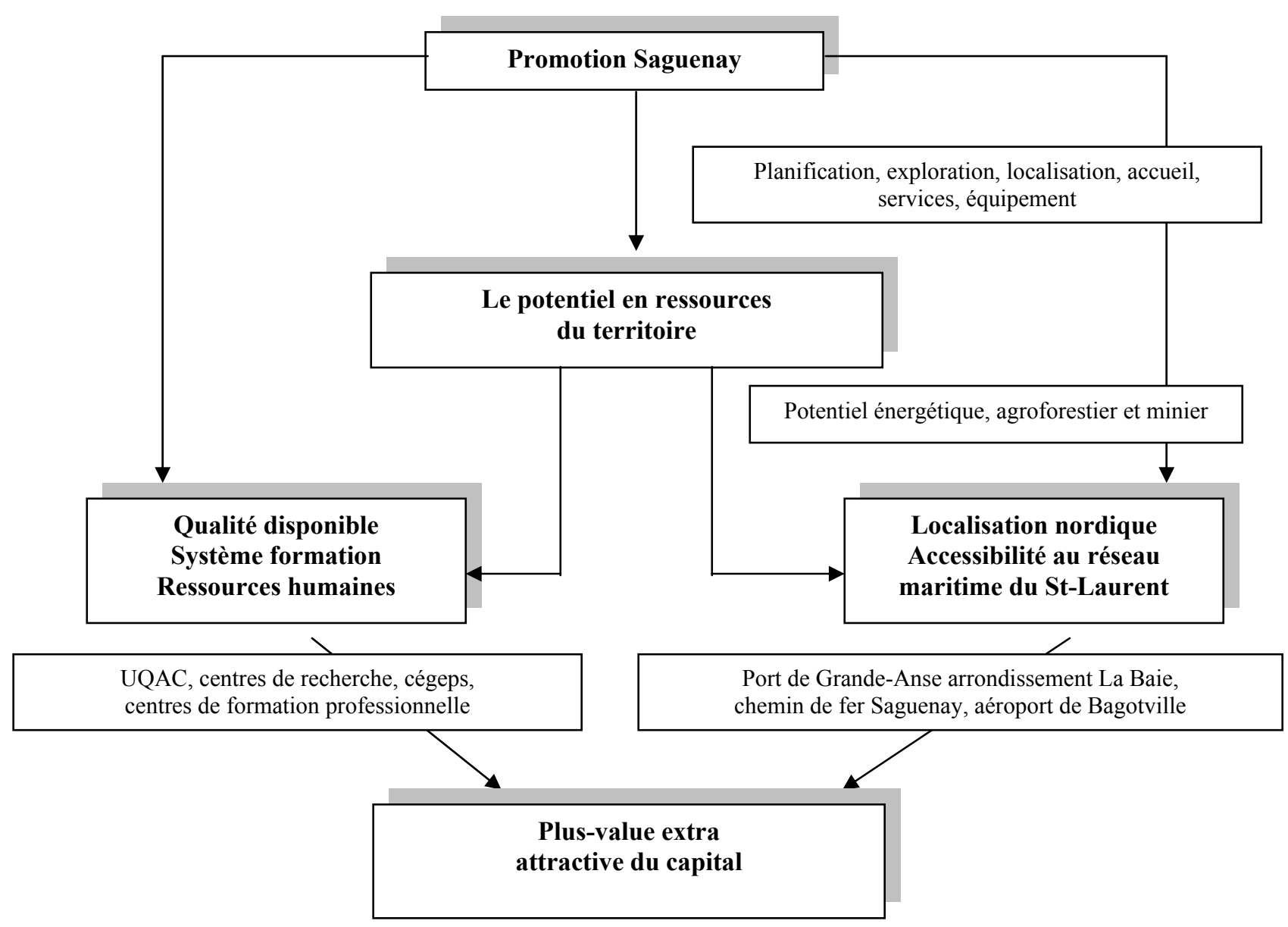

Écart entre le rendement et le coût de reproduction de la force du travail (logement, nourriture, transport, loisirs)

\section{Conclusion}

La création au Saguenay d'une base de l'offre constitue la condition première à l'implantation d'un pôle de compétitivité permettant de réaliser des gains continus sur les échanges avec le reste du monde (tableau $1)$.

Ces gains déterminent toute la dynamique cumulative du développement que Saguenay peut réaliser en utilisant deux leviers complémentaires :
- les leviers de la mondialisation en termes de capacité d'insertion dans les réseaux mondiaux des échanges et/ou l'attraction de flux du capital à la recherche de lieux optimaux de localisation;

- les leviers propres à la métropole économique d'une région ayant une dotation favorable en ressources et une localisation appropriée dans l'espace reliant le Moyen-Nord au marché continental (ALENA) du nord-est américain. 


\section{Tableau 1 - Ventilation des investissements publics requis par la ville de Saguenay sur cinq ans}

Infrastructure industrielle :

- Aménagement du complexe portuaire de Grande-Anse

40 millions\$

- Jonction Roberval Saguenay-Grande-Anse

10 millions\$

- Aménagement du nouveau parc industriel

10 millions\$

Sous-total :

60 millions\$

- Formation force de travail qualifié et / ou spécialisé

10 millions\$

- Apprentissages technologiques nouvelles et rétro ingénierie

10 millions\$

- Exploration des marchés et promotion mondiale du pôle de Saguenay

5 millions\$

- Services industriels (eau, gaz, énergie, traitement déchets industriels)

10 millions\$

- Subventions et services aux nouvelles firmes

30 millions\$

Sous-total :

65 millions\$

- Redressement démographique et stratégie d'attraction de 1000 immigrants qualifiés $(20000 \$$ x 1000$)$

20 millions\$

Aménagements urbains, services de logements et loisirs

5 millions\$

Sous-total :

25 millions\$

Total (soit 30 millions\$ par année) :

150 millions\$

La variante déterminante à la création d'une impulsion soutenue de développement se résume dans l'effort d'investissement à mobiliser pour établir une base appropriée de l'offre. Un estimé préliminaire (tableau 2) de l'effort financier à consacrer pour créer une base appropriée se chiffre à environ un milliard de dollars en cinq ans (200 millions de dollars par année en moyenne) à consacrer aux différents éléments qui forment la base territoriale de l'offre selon les économistes de l'Université de Berkley (Berkley Research Industrial Endowment), soit :

- les matériaux, composants des équipements requis pour le développement des produits;

- les technologies nouvelles à introduire;

- la configuration des échanges entre les firmes qui fournissent ou utilisent ces éléments dans le cadre de grappes industrielles à constituer.
De toute évidence, le contexte budgétaire actuel exclut toute possibilité de financement à partir de la contribution du Trésor public québécois, y compris le palier fédéral. À cette fin, nous avons établi une possibilité concrète de financement que Saguenay peut utiliser dans le cadre d'une transformation institutionnelle consacrant l'autonomie décisionnelle et fiscale ${ }^{6}$ de la métropole régionale.

Cette option comporte deux sources distinctes :

- la récupération d'une partie de la rente générée par l'exploitation des ressources relevant de la Couronne, soit au minimum 80 à 100 millions de dollars par année;

- un remboursement accéléré des reports d'impôts consentis aux firmes opérant dans le secteur des ressources. Il s'agit d'un minimum de 50 millions de dollars par année. Pour la multinationale Alcan, la somme des reports se chiffrait à $1180000000 \$$ en 2000. 


\section{Tableau 2 - Estimé de l'investissement en capital requis pour l'implantation d'un pôle de compétitivité au Saguenay}

\section{Nouvelles activités industrielles à localiser :}

Filière agroalimentaire

- Implantation de silos à grains (Grande-Anse)

- Production d'insuline, enzymes industriels

- Produits pharmaceutiques industriels

Filière du bois, papiers, produits dérivés

Portes, châssis, meubles

Filière métallique

Estimé des investissements privés requis et réalisables en cinq ans

Investissements publics requis en infrastructures et services par la ville de Saguenay

\section{Technologies requises :}

- Entreposage, nettoyage des grains

- Lait, viande porcine, malt

- Purification, réfrigération

- Chimie industrielle liée, machines et équipements forestiers

- Pièces d'aluminium et composants de l'industrie automobile

- Équipement hospitalier (appareils et instruments de précision)

- Composants et équipements aéronautiques

800 millions\$ à 1 milliard\$ en 5 ans, soit 200 millions\$ par an

150 à 200 millions\$, soit 40 millions\$ par an
Sans parler des possibilités de récupération d'une part du surplus fiscal que le Trésor public réalise au Saguenay-Lac-St-Jean, soit 336 M\$ par an. Saguenay peut récupérer $56 \%$ de ce surplus, soit $170 \mathrm{M} \$$ environ. Par conséquent, une réforme appropriée et équitable de la fiscalité provinciale fournirait les moyens requis au développement de Saguenay. À défaut de cette transformation réalisable ${ }^{7}$, les discours qu'entretiennent les tenants du développement par petites doses, ou ceux qui misent sur l'entrepreneuriat, ne peuvent conduire qu'à des gains marginaux ou à une trajectoire de similitude améliorée. Le développement de la nouvelle métropole requiert une mutation institutionnelle attribuant deux instruments stratégiques à Saguenay, soit :

- une autonomie décisionnelle en matière de stratégies de développement réalisables;

- des instruments réglementaires, budgétaires et fiscaux requis pour soutenir toute impulsion durable de développement ${ }^{8}$.

\section{Notes et références}

1 Sergieh F. Moussally est détenteur d'un doctorat en économie de l'Université de Neuchâtel et professeur titulaire à
l'Université du Québec à Chicoutimi. Les travaux du chercheur portent sur l'autonomie décisionnelle et fiscale des entités régionales et la mondialisation. Les textes de Monsieur Moussally sont disponibles à l'adresse électronique suivante : www.saguenaylacstjean.ca, section documents.

2 Porter, M.E. (2000). Locations, Clusters and Company Strategy, in Economic Geography, London, Oxford University Press, p. 253-274.

3 Notion conventionnelle de handicap au développement: taille du marché, localisation périphérique, spécialisation industrielle, disponibilité du capital, retard technologique, etc.

4 Aydalot, P. (dir.) (1986). Milieux innovateurs en Europe. Paris, GREMI.

5 Castells, Manuel (1998). La société en réseau, Paris, Fayard.

6 Moussally, Sergieh, F. (2000), L'autonomie décisionnelle et fiscale, Chicoutimi, Université du Québec à Chicoutimi.

7 Moussally, Sergieh, F. (2002), La transformation des assises économiques des régions ressources du Québec, Chicoutimi, Université du Québec à Chicoutimi.

8 Moussally, Sergieh, F. (2002), Le renouveau du fédéralisme canadien et le développement des régions du Québec, Saguenay, août. 